Commun. math. Phys. 2, 375-383 (1966)

\title{
Analytic Properties of Scattering Amplitudes in two Variables in General Quantum Field Theory
}

\author{
H. LEHMANN* \\ Institut des Hautes Etudes Scientifiques \\ Bures-sur-Yvette, (Seine-\&-Oise), France
}

Received February 21, 1966

\begin{abstract}
It is shown that scattering amplitudes have analytic properties as functions of momentum transfer not only for physical, but also for complex energies. This follows from local relativistic field theory for all reactions for which an ordinary dispersion relation can be proved.

It is further shown that such amplitudes are boundary values of analytic functions of the two variables energy and momentum transfer. A domain of holomorphy - which is however not best possible - is given explicitly. It follows then that partial waves can be analytically continued to complex energies.
\end{abstract}

\section{$\$ 1$ Introduction}

As is well known, analytic properties of scattering amplitudes as functions of energy or momentum transfer can be deduced from local relativistic field theory provided that certain mass-spectrum conditions are satisfied. HEPP [1] has given a complete mathematical derivation of these properties. Much less is known in this framework about analyticity in both variables simultaneously. MandeLstam [2] obtained a first result of this type. His method is applicable only in cases where ordinary dispersion relations can be derived for all three channels of a reaction. This includes $\pi-\pi$ and $\pi-K$ scattering [3] but not $\pi-N$ scattering. More recently, Bros, Epstein and GLASER [4], [5] proved that for a general two-particle reaction (excluding zero-mass particles) the amplitude is the boundary value of an analytic function of two variables which is holomorphic in a complex neighborhood of all physical points and the different channels are related by crossing-symmetry.

In the following we determine explicitly a complex domain in which the analytically continued amplitude is holomorphic in both variables for any scattering process for which an ordinary (energy) dispersion relation follows from field theory. We show further that this implies some analytic structure of the physical partial waves. These results are obtained by

* Permanent address: University of Hamburg, Germany. 
combining known one-variable properties of the amplitude without leaving the mass-shell.

We consider the fact interesting that the known one-variable properties on the mass-shell - dispersion relation in energy and analyticity in momentum transfer ellipses - are not saturated but lead necessarily to further properties of the scattering amplitude. The problem of displaying these further properties is only partially solved in this paper. The domain of holomorphy that we obtain explicitly is for this reason far from being best possible. It is therefore not surprising that - if one specializes to the $\pi-\pi$ system and compares our results with those of MANDELSTAM [2], who used a very different but also incomplete method - neither result contains the other [6].

\section{$\$ 2$. Analyticity in energy and momentum transfer}

Consider an elastic scattering process of scalar particles with masses $m$ and $\mu$. Let

$$
s=s_{1}+i s_{2}, \quad t=t_{1}+i t_{2}, \quad u=2 m^{2}+2 \mu^{2}-s-t
$$

be the usual Mandelstam variables such that $s$ and $-t$ correspond to the squares of c.m. energy and momentum transfer. We restrict ourselves to processes with a mass spectrum such that the following properties of the scattering amplitude $T\left(s_{1}, t_{1}\right)$ can be derived from field theory:

(A) A dispersion relation holds for fixed $t_{0}<t=t_{1} \leqq 0$; i.e. the physical amplitude $T\left(s_{1}, t_{1}\right)$ is the boundary value of an analytic function

$$
T\left(s, t_{1}\right)=T\left(u, t_{1}\right)=T^{*}\left(s^{*}, t_{1}\right)
$$

holomorphic in the $s$-plane except for poles related to stable particles and cuts along

$$
s=s_{1} \geqq(m+\mu)^{2} \quad \text { and } \quad s=s_{1} \leqq(m-\mu)^{2}-t_{1} .
$$

We assume for simplicity $\left|t_{0}\right|<4 \mu m$ which is true for $\pi-N$ scattering. Also, we substract the pole terms and denote by $T$ the pole-free amplitude.

(B) For fixed $s=s_{1}>(m+\mu)^{2}$ the physical amplitude has an analytic continuation $T\left(s_{1}, t\right)$ in $t$ into the ellipse

with

$$
E\left(s_{1}\right)=\left\{|t|+\left|t+4 k^{2}\right|<4 k^{2} a\left(s_{1}\right)\right\}
$$

$$
a\left(s_{1}\right)=\left[1+\frac{\left(m_{1}^{2}-\mu^{2}\right)\left(m_{2}^{2}-m^{2}\right)}{k^{2}\left[s_{1}-\left(m_{1}-m_{2}\right)^{2}\right]}\right]^{\frac{1}{2}} .
$$

$k^{2}\left(s_{1}\right)$ is the square of c.m. momentum and $m_{1}, m_{2}$ are given for each process [7]; e.g. $m_{1}=3 \mu, m_{2}=m+\mu$ for $\pi-N$ scattering. 
Further properties of the functions appearing in (A) and (B) follow from the proof of these relations [1]. The following properties are sufficient for our purpose:

(C) The analytic function $T\left(s, t_{1}\right)$ is continuous in $\left(s, t_{1}\right)$ and bounded by a polynomial in $s$ for $s \rightarrow \infty$. The boundary values $T\left(s_{1}, t_{1}\right)$ are tempered distributions in $s_{1}$ and continuous in $t_{1}$. Also, $T\left(s_{1}, t\right)$ is a tempered distribution in $s_{1}$ for $t \in E\left(s_{1}\right)$.

We show that from (A), (B) and (C) a holomorphy domain in both variables follows. Actually we prove first that the $t$-analyticity expressed in (B) can be extended from physical $s$-values to the cut complex $s$-plane such that for any fixed $s, T\left(s, t_{1}\right)$ is in fact analytic in $t$ in a domain which includes the interval $t_{0}<t=t_{1} \leqq 0$. The domain which we obtain by our method is not best possible. Our result follows already if we replace $(\mathrm{B})$ by the weaker statement:

$\left(B_{\alpha}\right)$ For fixed $s=s_{1}>s_{1 \alpha}>(m+\mu)^{2}$ the physical amplitude has an analytic continuation $T\left(s_{1}, t\right)$ into $t \in E_{\alpha}\left(s_{1}\right)$ which is the largest ellipse with foci at $t=0$ and $t=\alpha t_{0}(0<\alpha<1)$ which can be inscribed into $E\left(s_{1}\right)$. Explicitly:

with

$$
E_{\alpha}\left(s_{1}\right)=\left\{|t|+\left|t-\alpha t_{0}\right|<\alpha\left|t_{0}\right| a_{\alpha}\left(s_{1}\right)\right\}
$$

$$
a_{\alpha}\left(s_{1}\right)= \begin{cases}\frac{4 k^{2}}{\alpha\left|t_{0}\right|}(a+1)-1 & \text { for } \frac{2}{a+1}<\frac{4 k^{2}}{\alpha\left|t_{0}\right|}<1 \\ \frac{4 k^{2}}{\alpha\left|t_{0}\right|}(a-1)+1 & \text { for } \frac{4 k^{2}}{\alpha\left|t_{0}\right|}>1 .\end{cases}
$$

The ellipses $E_{\alpha}\left(s_{1}\right)$ are easier to handle since their foci are independent of $s_{1}$ and lie in the $t$-interval for which the dispersion relation (A) holds.

We prove now the following

Theorem: For any $s$ in the complex $s$-plane with cuts along

$$
s=s_{1} \geqq(m+\mu)^{2} \text { and } s=s_{1} \leqq(m-\mu)^{2}-\alpha t_{0},
$$

$T\left(s, t_{1}\right)$ has an analytic continuation $T(s, t)$ which is holomorphic in $t$ for $t \in \in_{0<\alpha<1} E_{\alpha}(s)$.

where

$$
E_{\alpha}(s)=\left\{|t|+\left|t-\alpha t_{0}\right|<\alpha\left|t_{0}\right| a_{\alpha}(s)\right\}
$$

and

$$
a_{\alpha}(s)=\frac{1}{2}\left(\left|R_{\alpha}(s)\right|+\left|R_{\alpha}(s)\right|^{-1}\right)
$$

$$
\begin{aligned}
R_{\alpha}(s)=\exp & \left\{\frac{1}{i \pi}\left[s-(m+\mu)^{2}\right]^{\frac{1}{2}}\left[s-(m-\mu)^{2}+\alpha t_{0}\right]^{\frac{1}{2}} \times\right. \\
& \left.\times \int_{s_{1 \alpha}}^{\infty} \frac{d s_{1}^{\prime} \log \left|R_{\alpha}\left(s_{1}^{\prime}\right)\right|}{\left[s_{1}^{\prime}-(m+\mu)^{2}\right]^{\frac{1}{2}}\left[s_{1}^{\prime}-(m-\mu)^{2}+\alpha t_{0}\right]^{\frac{1}{2}}} \cdot \frac{1}{s_{1}^{\prime}-s}\right\}
\end{aligned}
$$


$T(s, t)$ is an analytic function of the two complex variables $s, t$ which is holomorphic in $G=\bigcup_{0<\alpha<1} G_{\alpha}$ with

$G_{\alpha}=\left\{(s, t): s \notin s_{1} \geqq(m+\mu)^{2} ; s \notin s_{1} \leqq(m-\mu)^{2}-\alpha t_{0} ; t \in E_{\alpha}(s)\right\}$

Remark: $\log \left|R_{\alpha}(s)\right|$ is the harmonic function in the cut $s$-plane with boundary values

$\log \left|R_{\alpha}\left(s_{1}\right)\right|=\left\{\begin{array}{l}\log \left(a_{\alpha}\left(s_{1}\right)+\left[a_{\alpha}^{2}\left(s_{1}\right)-1\right]^{\frac{1}{2}}\right) \text { for } s_{1}>s_{1 \alpha} \\ 0 \text { for }(m+\mu)^{2} \leqq s_{1} \leqq s_{1 \alpha} \text { and } s_{1} \leqq(m-\mu)^{2}-\alpha t_{0} .\end{array}\right.$

The minimum principle for harmonic functions implies $\left|R_{\alpha}(s)\right|>1$ everywhere in the cut $s$-plane, so that the domain of $t$-holomorphy is non-empty for all $s$ not on the left-hand cut.

We first prove this theorem under the assumption that $T\left(s_{1}, t_{1}\right)$ is a continuous function of $s_{1}, t_{1}$. The case of distributions can then be treated by a suitable regularization.

Let

\section{a) Continuous boundary values}

$$
c_{n}(s)=\frac{1}{\alpha t_{0}} \int_{0}^{\alpha t_{0}} d t_{1} T\left(s, t_{1}\right) P_{n}\left(1-\frac{2 t_{1}}{\alpha t_{0}}\right)
$$

be the Legendre coefficients of the analytic function $T\left(s, t_{1}\right)$ referred to in (A). With our continuity assumption, $c_{n}(s)$ is an analytic function in the whole $s$-plane, except for the cuts (8), with continuous boundary values, bounded by a polynomial and by

$$
\left|c_{n}(s)\right| \leqq \operatorname{Max}_{\alpha t_{0} \leqq t_{1} \leqq 0}\left|T\left(s, t_{1}\right)\right| \text {. }
$$

We conclude from $\left(B_{\alpha}\right)$ - using a standard theorem on Legendre series which represent functions analytic in ellipses,

for any

$$
\left|c_{n}\left(s_{1}\right)\right| \leqq \frac{c\left(s_{1}\right)}{\varrho_{\alpha}^{n}\left(s_{1}\right)}
$$

$$
\varrho_{\alpha}\left(s_{1}\right)<\left|R_{\alpha}\left(s_{1}\right)\right|=a_{\alpha}\left(s_{1}\right)+\left[a_{\alpha}^{2}\left(s_{1}\right)-1\right]^{\frac{1}{2}} .
$$

Combining (15) and (16) with the definition

$$
\varrho_{\alpha}\left(s_{1}\right)=1 \text { if }(m+\mu)^{2} \leqq s_{1} \leqq s_{1 \alpha} \text { and } s_{1}<(m-\mu)^{2}-\alpha t_{0}
$$

we have everywhere on the boundary the inequality

$$
\left|c_{n}\left(s_{1}\right)\right| \leqq \frac{c\left(s_{1}\right)}{\varrho_{\alpha}^{n}\left(s_{1}\right)}
$$

with $c\left(s_{1}\right)$ bounded by a polynomial of some degree $M$, since both $T\left(s, t_{1}\right)$ and $T\left(s_{1}, t\right)$ have a polynomial bound. 
To obtain functions bounded at infinity, we divide by a suitable function and define the quantities

$$
\begin{aligned}
d_{n}(s)= & \left.\frac{c_{n}(s)}{\left[\frac{s-(m+\mu)^{2}}{s-(m-\mu)^{2}+\alpha t_{0}}\right]^{\frac{1}{2}}-1}-i \gamma\right\}^{M} \times \\
& \times\left\{\frac{1}{\left[\frac{s-(m+\mu)^{2}}{s-(m-\mu)^{2}+\alpha t_{0}}\right]^{\frac{1}{2}}+1}+i \gamma\right\}^{M} \\
& \left(\gamma>0, \operatorname{Im}\left[\frac{s-(m+\mu)^{2}}{s-(m-\mu)^{2}+\alpha t_{0}}\right]^{\frac{1}{2}} \geqq 0\right)
\end{aligned}
$$

which have the same analytic properties and satisfy on the cuts

$$
\left|d_{n}\left(s_{1}\right)\right| \leqq \frac{d}{\varrho_{\alpha}^{n}\left(s_{1}\right)}
$$

where $d$ is independent of $n$ and $s_{1}$.

We then introduce

$$
e_{n}(s)=d_{n}(s) r_{\alpha}^{n}(s)
$$

where $r_{\alpha}(s)$ is the bounded function with boundary values $\left|r_{\alpha}\left(s_{1}\right)\right|$ $=\varrho_{\alpha}\left(s_{1}\right)$ which is analytic and without zeros in the cut $s$-plane.

Explicitly

$$
\begin{aligned}
r_{\alpha}(s)=\exp & \left\{\frac{1}{i \pi}\left[s-(m+\mu)^{2}\right]^{\frac{1}{2}}\left[s-(m-\mu)^{2}+\alpha t_{0}\right]^{\frac{1}{2}} \times\right. \\
& \left.\times \int_{s_{1 \alpha}}^{\infty} \frac{d s_{1}^{\prime} \log \varrho_{\alpha}\left(s_{1}^{\prime}\right)}{\left[s_{1}^{\prime}-(m+\mu)^{2}\right]^{\frac{1}{2}}\left[s_{1}^{\prime}-(m-\mu)^{2}+\alpha t_{0}\right]^{\frac{1}{2}}} \cdot \frac{1}{s_{1}^{\prime}-s}\right\}
\end{aligned}
$$

$e_{n}(s)$ is a bounded analytic function which satisfies

$$
\left|e_{n}(s)\right| \leqq d
$$

in the entire $s$-plane. This follows from the bound on the cuts by the maximum principle.

Therefore

$$
\left|d_{n}(s)\right| \leqq \frac{d}{\left|r_{\alpha}(s)\right|^{n}}
$$

and consequently, for arbitrary $s$,

$$
\begin{aligned}
\left|c_{n}(s)\right| \leqq\left|\frac{1}{\left[\frac{s-(m+\mu)^{2}}{s-(m-\mu)^{2}+\alpha t_{0}}\right]^{\frac{1}{2}}-1}-i \gamma\right|^{M} \times \\
\times\left|\frac{1}{\left[\frac{s-(m+\mu)^{2}}{\left.s-(m-\mu)^{2}+\alpha t_{0}\right)}\right]^{\frac{1}{2}}+1}-i \gamma\right|^{M} \cdot \frac{d}{\left|r_{\alpha}^{n}(s)\right|}
\end{aligned}
$$


This formula exhibits an exponential decrease of $\left|c_{n}(s)\right|$ as a function of $n$ which ensures the convergence of

$$
T(s, t)=\sum_{n}(2 n+1) c_{n}(s) P_{n}\left(1-\frac{2 t}{\alpha t_{0}}\right)
$$

for complex $t \in E_{\alpha}(s)$.

We can then take the union of the ellipses $E_{\alpha}(s)$ over the interval $0<\alpha<1$.

Finally, we conclude from $(26)$ and $(27)$ that $T(s, t)$ is an analytic function of $s$ and $t$ since the series (27) converges uniformly in the domain $G_{\alpha}$ given in the theorem.

\section{b) Distributions}

In general, the boundary values of $T\left(s, t_{1}\right)$ may be tempered distributions. We indicate how this case can be reduced to the case of continuous boundary values.

Define the regularized function

$$
\left.T_{\varphi}\left(s, t_{1}\right)\right)=\int d s_{1}^{\prime} \varphi\left(s_{1}-s_{1}^{\prime}\right) T\left(s_{1}^{\prime}+i s_{2}, t_{1}\right)
$$

where $\varphi$ is infinitely differentiable and $\varphi(\xi)=0$ for $|\xi|>\varepsilon$. By standard theorems, $T_{\varphi}$ has for real $s$ the desired continuity properties; ([8], p. $82 \mathrm{ff}$.).

On the other hand, it is easily checked that the properties (A) and $\left(\mathrm{B}_{\alpha}\right)$ hold for $T$ with the following modifications:

(A) $T_{\varphi} T_{\varphi}\left(s, t_{1}\right)$ is analytic in the $s$-plane with cuts

$$
s=s_{1} \geqq(m+\mu)^{2}-\varepsilon \text { and } s=s_{1} \leqq(m-\mu)^{2}-\alpha t_{0}+\varepsilon .
$$

$\left(\mathrm{B}_{\alpha}\right)_{\varphi} T_{\varphi}\left(s_{1}, t\right)$ is analytic in $t \in E_{\alpha, \varepsilon}\left(s_{1}\right)$, with

and

$$
E_{\alpha, \varepsilon}\left(s_{1}\right)=\left\{|t|+\left|t-\alpha t_{0}\right|<\alpha\left|t_{0}\right| a_{\alpha, \varepsilon}\left(s_{1}\right)\right\}
$$

$$
a_{\alpha, \varepsilon}\left(s_{1}\right)=\operatorname{Min}_{s_{1}-\varepsilon \leqq s_{1}^{\prime} \leqq s_{1}+\varepsilon} a_{\alpha}\left(s_{1}^{\prime}\right) .
$$

Therefore the preceding argument applies to the coefficients

$$
c_{n, \varphi}(s)=\frac{1}{\alpha t_{0}} \int_{0}^{\alpha t_{0}} d t_{1} T_{\varphi}\left(s_{1}, t_{1}\right) P_{n}\left(1-\frac{2 t_{1}}{\alpha t_{0}}\right)
$$

with the result that

and that

$$
\left|c_{n, \varphi}(s)\right| \leqq \frac{c_{\varphi}(s)}{\left|r_{\alpha, \varepsilon}(s)\right|^{n}}
$$

$$
T_{\varphi}(s, t)=\sum_{n=0}^{\infty}(2 n+1) c_{n, \varphi}(s) P_{n}\left(1-\frac{2 t}{\alpha t_{0}}\right)
$$

is holomorphic in $G_{\alpha, \varepsilon}$.

Furthermore

$$
c_{n, \varphi}(s)=\int d s_{1}^{\prime} \varphi\left(s_{1}-s_{1}^{\prime}\right) c_{n}\left(s_{1}^{\prime}+i s_{2}\right)=\left(\varphi * c_{n}\right)(s)
$$


satisfies the relation

$$
c_{n, \varphi}(s)=c_{n, \varphi_{x}}(s-x) ; \varphi_{x}(\xi)=\varphi(\xi+x) .
$$

For suitable $x$ and support of $\varphi$ this leads to

In particular, we have

$$
T_{\varphi}(s, t)=T_{\varphi_{x}}(s-x, t) .
$$

The distribution

$$
T_{\varphi}(s, t)=T_{\varphi_{s_{1}}}\left(i s_{2}, t\right)
$$

is of the form

$$
\begin{aligned}
T_{\varphi}(\psi) & =\int d s_{1} d s_{2} d t_{1} d t_{2} \psi\left(s_{1}, s_{2}, t_{1}, t_{2}\right) T_{\psi}(s, t) \\
& =\int d s_{1} d s_{2} d t_{1} d t_{2} \psi\left(s_{1}, s_{2}, t_{1}, t_{2}\right) T_{\varphi_{s_{1}}}\left(i s_{2}, t\right)
\end{aligned}
$$

with

$$
T_{\varphi}(\psi)=V(\varphi * \hat{\psi})
$$

where $V(\psi)$ is the distribution related to $T_{\varphi}\left(i s_{2}, t\right)$ by the nuclear theorem.

Let $\left\{\varphi_{\nu}(\xi)\right\}$ be a sequence which approaches $\delta(\xi)$. Then for all $\psi$ with suitable support

$$
T_{\varphi_{\nu}}(\psi)=V\left(\varphi_{\nu} * \hat{\psi}\right) \rightarrow V(\hat{\psi})
$$

and, since $T_{\varphi_{\nu}}(s, t)$ is a convergent sequence of holomorphic functions for all test functions, it converges to a holomorphic function $T(s, t)$. Therefore we have

$$
V(\hat{\psi})=T(\psi)=\int d s_{1} d s_{2} d t_{1} d t_{2} \psi\left(s_{1}, s_{2}, t_{1}, t_{2}\right) T(s, t) .
$$

We obtain in this way the result that the scattering amplitude has an analytic continuation $T(s, t)$ which is holomorphic in the domain $G$ given in the theorem, also for distribution boundary values.

\section{§ 3. Partial waves}

The analytic properties of the amplitude as a function of two complex variables make it possible to analytically continue the physical partial waves to complex energies. We discuss this briefly. Define partial waves by

$$
t_{l}(s)=\frac{1}{k^{2}(s)} \int_{-4 k^{2}(s)}^{0} d t T(s, t) P_{l}\left(1+\frac{t}{2 k^{2}(s)}\right) .
$$

These are physical quantities for $s=s_{1} \geqq(m+\mu)^{2}$.

For complex $s$ we keep the relation

$$
k^{2}(s)=\frac{\left[s-(m+\mu)^{2}\right]\left[s-(m-\mu)^{2}\right]}{4 s}
$$

and integrate along a straight line in the $t$-plane. The partial waves $t_{l}(s)$ 
can be continued from physical values of $s$ to complex values which are determined by the condition that the whole path of the $t$-integration in (44) lies in the domain of holomorphy $G$ of $T(s, t)$. It is easily checked that this is the case if the endpoint obeys the relation

$$
t=-4 k^{2}(s) \in \bigcup_{0<\alpha<1} E_{\alpha}(s) .
$$

For a fixed value of $\alpha$ this condition, according to (9), is equivalent to

$$
4\left|k^{2}(s)\right|+\left|4 k^{2}(s)+\alpha t_{0}\right|<\alpha\left|t_{0}\right| a_{\alpha}(s) .
$$

This determines a finite domain in $s$-space, whose exact shape is not easily calculable. For physical $s$-values (46) gives - after taking the union over $\alpha$ - the interval

$$
0<k^{2}\left(s_{1}\right)<\frac{\left|t_{0}\right|}{2\left(3-a\left(s_{1}\right)\right)},
$$

where the partial waves are boundary values of analytic functions whose domain of holomorphy consists of the points which satisfy (46), are not on the $s$-cuts and are connected with the interval (47).

\section{\$ 4. Crossing symmetry and further problems}

We pointed out already that our treatment is incomplete in several respects. One property which we did not make use of so far is $s, u$ crossing symmetry as expressed by $T\left(s, t_{1}\right)=T\left(u, t_{1}\right)$. This can be taken into account by applying the procedure used in $\S 2$ to $T(u, t)$, which is holomorphic in the cut $u$-plane if $t_{0}<t=t_{1}<0$ and holomorphic in $t \in E\left(u_{1}\right)$ if $u=u_{1}>(m+\mu)^{2}$. Therefore, by substituting $u$ for $s$ in all relations, we obtain a new domain $G^{\prime}$ of holomorphy of $T(s, t)$ if we replace $s$ by $u=2 m^{2}+2 \mu^{2}-s-t$ in eq. (12). $T(s, t)$ is then holomorphic in the union of $G$ and $G^{\prime}$.

Other shortcomings of our method are not so easily overcome. A severe limitation is of course the replacement of the ellipses $E\left(s_{1}\right)$ by $E_{\alpha}\left(s_{1}\right)$. This is a drastic step for large values of $s_{1}$ and has the consequence that we cannot show - as Bros, EPstein and Glaser did by other methods - that the physical amplitude is at all points a boundary value of an analytic function of $s$ and $t$.

A further problem - which has been partially treated by PoHLMEYER [6] - is to take into account the fact that the imaginary part of the amplitude has a larger domain of analyticity in momentum transfer. This should lead to an essential improvement, particularly for low energies.

Acknowledgements. The author wishes to thank Drs. H. J. Borchers, J. LAScoux and D. Ruelle for helpful discussions. He is grateful to Professor L. MotCHANE for hospitality at the Institut des Hautes Etudes Scientifiques. 


\section{References}

[1] Hepp, K.: Helv. phys. Acta 37, 639 (1964).

[2] Mandelstam, S.: Nuovo Cimento 15, 658 (1960).

[3] JIN, Y. S.: Nuovo Cimento 25, 1356 (1962).

[4] Bros, J., H. Epstein, and V. Glaser: Nuovo Cimento 31, 1256 (1964).

[5] - Commun. math. Phys. 1, 240 (1965).

[6] Pohlmeyer, K.: Diplomarbeit Hamburg, 1964; unpublished.

[7] Lehmann, H.: Nuovo Cimento 10, 594 (1958).

[8] Streater, R. F., A. S. Wightman: PCT, spin and statistics and all that. New York: W. A. Benjamin, Inc. 1964. 